\title{
ESTUDIOS DE HIPERCOAGULABILIDAD EN OFTALMOLOGÍA. ¿QUÉ PEDIR Y CUÁNDO?
}

\section{HYPERCOAGULABLE WORKUP IN OPHTHALMOLOGY. WHEN AND WHAT?}

\author{
MUÑOZ-NEGRETE FJ ${ }^{1}$, CASAS-LLERAS P², PÉREZ-LÓPEZ M², REBOLLEDA G ${ }^{1}$
}

\begin{abstract}
RESUMEN
La mayoría de los trastornos oftalmológicos secundarios a hipercoagulabilidad se deben a la confluencia de factores congénitos y adquiridos. Dada la multitud de test diagnósticos existentes se hace preciso una sistematización de la solicitud de los mismos. La mayoría de los trastornos congénitos de la coagulación producen trombosis venosas y son de herencia autosómica dominante. Los más frecuentes son por este orden la resistencia a la proteína $\mathrm{C}$ activada (Factor V Leiden), la mutación del gen de la protrombina (G20210A), déficit de proteína C proteína $\mathrm{S}$ y de antitrombina III. La anemia de células falciformes puede acompañarse de fenómenos oclusivos arteriales y venosos.

Respecto a las trombosis arteriales, los marcadores implicados más frecuentemente son los niveles de homocisteína en ayunas y los del síndrome de anticuerpos antifosfolípidos, aunque ambos producen oclusiones venosas fundamentalmente.

Diversos factores adquiridos pueden producir estados hipercoagulables. Dentro de las entidades más frecuentes destacamos la hiperhomocisteinemia y el síndrome de anticuerpos antifosfolípidos, sin olvidar múltiples circunstancias como patología hepática, ingesta de alcohol, tabaco, anticonceptivos orales, inmovilización, cirugía y enfermedades mielo-
\end{abstract}

\footnotetext{
Recibido: 13/5/09. Aceptado: 15/7/09.

Servicio de Oftalmología. Hospital Ramón y Cajal. Madrid. España.

1 Doctor en Medicina.

2 Licenciado en Medicina.

Correspondencia:

Francisco J. Muñoz-Negrete

Servicio de Oftalmología. Hospital Ramón y Cajal

Carretera Colmenar Viejo, km. 9,1

28034 Madrid

España

E-mail: franciscojmunoz@telefonica.net
}

Most ophthalmologic disorders secondary to hypercoagulabe state are due to the confluence of congenital and adquired factors. A systematic workup is mandatory.

Most of congentital coagulation disorders cause venous trombosis and are inherited autosomal dominantly. In order of frequency these are factor $\mathrm{V}$ Leiden mutation (activated protein $\mathrm{C}$ resistance), G20210A mutation of the prothrombin gen and protein $\mathrm{C}$, protein $\mathrm{S}$, and antithrombin III deficiencies. Sickle cell anemia can determine arerial and venous thrombosis.

In relation with arterial occlusion, the markers most frequently involved are homcysteine fasting levels and the markers of antiphospholipid antibody syndrome. Both of them can also determine venous thrombosis.

Several acquired factors can lead to hypoercoagulable state, especially hyperhomocysteinemia, antiphospholipid antibody syndrome, hepatic disease, alcohol and tobacco intake, oral contraceptives, immobilization, surgeries and malignancies.

In central venous occlusion is only necessary to rule out hyperhomocysteinemia and antiphospholipid antibody syndrome in young patients without known risk factors. 
proliferativas que pueden potenciar su desarrollo. En la oclusión de vena central de la retina sólo se indica descartar hiperhomocisteinemia y síndrome de anticuerpos antifosfolípidos en pacientes jóvenes sin factor de riesgo conocido.

En la embolia de la arteria central de la retina, sólo se recomienda estudio en menores de 50 años sin origen de émbolo detectable (jóvenes con alto riesgo). En este caso se investigará: proteína $\mathrm{C}, \mathrm{S}$, antitrombina III, homocisteína, electroforesis de hemoglobina y síndrome antifosfolípido.

En la neuropatía óptica isquémica no arterítica no se precisan estudios de hipercoagulabilidad.

Ante una amaurosis fugax sin fuente embolígena conocida se recomienda la búsqueda de alteraciones relacionadas con oclusiones arteriales, fundamentalmente déficit de antitrombina III, hiperhomocisteinemia, síndrome antifosfolípido y la enfermedad de células falciformes.

Palabras clave: Hipercoagulabilidad, oclusión vena central de la retina, embolia arteria central de la retina, hiperviscosidad.
In central artery occlusion, hypercoagulable workup is only recommended for patients less than 50 years-old with unknown emboli source. In this cases protein $\mathrm{C}$, protein $\mathrm{S}$, and antithrombin III deficiencies, homocystein, sickle cell diseae and antiphospholipid antibody syndrome will ruled out. In non arteritic ischemic optic neuropathy hypercoagulable work up is not necessary.

In amaurosis fugax without known emboli source, it is recommended to rule out etiologies of arterial occlusion, especially antithrombin III deficiencies, homocystein, sickle cell diseae and antiphospholipid antibody syndrome (Arch Soc Esp Oftalmol 2009; 84: 325-332).

Key words: Hypercoagulability, retinal venous occlusion, central retinal artery occlusion, hyperviscosity.
La hemostasia es un mecanismo fisiológico que mantiene la sangre en estado líquido.

Hablamos de estados de hipercoagulabilidad para referirnos a aquellas situaciones en las que existe un desequilibrio entre los factores pro-coagulantes y los anticoagulantes a favor de los primeros. Las manifestaciones derivarán de la perfusión anormal de los órganos y tejidos afectos.

Los trastornos de la coagulación pueden subdividirse en congénitos o adquiridos, aunque es frecuente que ambos tipos se combinen en el mismo paciente y ejerzan un efecto sinérgico (tabla I). Habitualmente se requiere la confluencia de múltiples factores de riesgo para que se produzcan fenómenos trombóticos.

\section{TRASTORNOS CONGÉNITOS}

Todos los trastornos congénitos de hipercoagulabilidad (tabla I) predisponen a trombosis venosas, si bien algunos también pueden predisponer a trombosis arteriales (mutación del gen de la protrombina G20210A, déficit de antitrombina III, síndrome de la plaqueta pegajosa y anemia de células falciformes) (1). La mayoría de ellos presentan una herencia autosómica dominante (AD).

\section{Resistencia a la Proteína C activada (Factor V Leiden)}

Se presenta en un 5\% en la población blanca, llegando hasta el $20 \%$ en Suecia y Oriente Medio. La herencia es AD. Se ha identificado en un $20 \%$ de los sujetos con un primer episodio de trombosis y en un 50\% con trombosis familiar. En el $95 \%$ de los casos el defecto se asocia a una sustitución de la arginina por la glicina en la posición 506 de la molécula del Factor V, causando una resistencia a la proteína $\mathrm{C}$ activada (PCA).

\section{Mutación del gen de la protrombina (G20210A)}

Presente en el 1-4\% de la población general. Se debe a una mutación ( $\mathrm{G}$ por $\mathrm{A}$ ) en el nucleótido 20210 del gen de la protrombina, que se asocia con un aumento de las concentraciones plasmáticas de protrombina. Aumenta el riesgo de trombosis venosa entre 3 y 5 veces, siendo mayor en aquellos que también son portadores del factor V Leiden (2). Se ha relacionado también con un mayor riesgo de accidente cerebro-vascular, infarto miocardio (afectación arterial) y oclusión de vena central de la retina (3). 
Tabla I. Causas de hipercoagulabilidad

\section{Factores congénitos}

- Resistencia a la Proteína C activada (FACTOR V Leiden)

- Mutación del gen de la protrombina (G20210A)

- Déficit de Proteína C

- Déficit de Proteína S

- Déficit de Antitrombina III

- Aumento de Factor VIII (> 1500 UI)

- Deficiencia del Cofactor II de la Heparina

- Disfibrogenemia

- Deficiencia congénita de plasminógeno

- Mutación de trombomodulina.

- Sticky platelet syndrome (síndrome de la plaqueta pegajosa

- Anemia de células falciformes

\section{Factores adquiridos}

- Patología hepática o endotelial.

- Déficit de vitamina C

- Anticonceptivos orales

- Alcohol

- Tabaco

- Situaciones especiales:

- Menopausia

- Embarazo

- Inmovilización

- Cirugía

- Traumatismos.

- Enfermedades:

- Cáncer, enfermedades mieloproliferativas,

- PTT

- Coagulación intravascular diseminada

- Sepsis

- Hiperhomocistinemia

- Síndrome de anticuerpos antifosfolípidos

\section{Déficit de Proteína C (0,15-0,5\% de la} población general, herencia $\mathrm{AD}$ )

Un $15 \%$ de los sujetos con déficit de proteína C que cursan con trombosis, presentan simultáneamente una resistencia a la PCA. Los anticumarínicos pueden inducir necrosis cutánea.

\section{Déficit de Proteína S (0,7\% de la población general, herencia AD)}

Al igual que el caso anterior, el defecto se encuentra en forma heterocigota en el 1-3\% de los sujetos con trombosis venosa. Se distinguen 3 tipos:

- Tipo I (Forma clásica) en la que existe un déficit cuantitativo y cualitativo
- Tipo II en el que únicamente hay alteración funcional de la proteína

- Tipo III en el que disminuye la proteína $S$ libre, aunque los valores de proteína $\mathrm{S}$ total pueden ser normales.

\section{Déficit de Antitrombina III $(0,17 \%$ de la población general, herencia $\mathrm{AD}$ )}

Los individuos heterocigotos tienen tasas de actividad funcional del 30 al $60 \%$ del valor normal, mientras que el estado homocigoto es teóricamente incompatible con la vida. Una variedad de este cuadro es la mutación del lugar de unión de la antitrombina III a la heparina. En este caso, la antitrombina mutada no puede unirse a la heparina disminuyendo su eficacia anticoagulante y de otros enzimas más proximales en la cascada de la coagulación. El resultado es un fenotipo que favorece la trombosis tanto arterial como venosa.

\section{Disfibrogenemia}

Causa rara de hipercoagulabilidad congénita. Se estima que se presenta en el $1 \%$ de sujetos jóvenes con trombosis de causa desconocida.

\section{Deficiencia congénita de plasminógeno}

Similar clínicamente al déficit de antitrombina III y proteína $\mathrm{C}$.

\section{Sticky platelet syndrome (síndrome de la plaqueta pegajosa)}

Da lugar a trombosis arteriales y venosas.

\section{Anemia de células falciformes $(8 \%$ afroamericanos heterocigotos y 1/600 afroamericanos homocigotos)}

Las mayores complicaciones derivan de la vasooclusión, tanto arterial como venosa, aunque principalmente de la primera. Puede afectar a todo tipo de vasos, preferentemente de pequeño calibre. 


\section{TRASTORNOS ADQUIRIDOS}

Además de las alteraciones congénitas de la coagulación, existen otros factores que pueden favorecer la hipercoagulabilidad:

1. Daño hepático o endotelial: Por ser origen de factores de la coagulación.

2. Déficit de sustratos: Déficit de vitamina C.

3. Anticonceptivos orales, alcohol, tabaco.

4. Situaciones especiales: Edad avanzada, menopausia, embarazo, inmovilización, cirugía, traumatismos.

5. Neoplasias, enfermedades mieloproliferativas, coagulación intravascular diseminada (CID), sepsis, síndromes de hiperviscosidad.

6. Hiperhomocistinemia

7. Síndrome de anticuerpos antifosfolípidos

\section{Hiperhomocisteinemia}

Influyen tanto factores genéticos como adquiridos. La homocisteína es un aminoácido derivado de la metionina que puede convertirse en cisteína, para ello se requiere la presencia de vitaminas B12, B6 y ácido fólico. Así, sujetos con mutaciones del enzima metilen-tetrahidrofolato-reductasa (MTHFR), cistationasa $ß$-sintetasa o con déficit de las vitaminas esenciales para su metabolismo, presentarán un acúmulo de homocisteína. Este aumento de homocisteína en sangre se ha asociado con trombosis venosa y enfermedad arterial (4).

\section{Anticuerpos antifosfolípidos}

La incidencia de anticuerpos antifosfolípidos en la población general es del 2-4\%. El síndrome clínico de anticuerpos antifosfolípidos incluye abortos de repetición, trombosis venosas y trombocitopenia. Dichos anticuerpos constituyen una familia heterogénea de anticuerpos con sensibilidad cruzada y se detectan por técnica ELISA. El anticoagulante lúpico prolonga las cascadas de la coagulación dependiente de los fosfolípidos. La prueba de anticoagulante lúpico es más específica de pacientes con riesgo de eventos tromboembólicos. Por el contrario, la prueba de anticuerpos anticardiolipina es más sensible pero inespecífica, pudiendo producir falsos positivos. El isotipo frecuentemente implicado en la trombosis es el IgG, específicamente el IgG2 (1).

\section{PANEL RECOMENDADO EN ESTADOS DE HIPERCOAGULABILIDAD}

Es muy importante realizar una completa anamnesis para valorar la existencia de factores de riesgo adquiridos, dado que la confluencia de diversos factores de riesgo congénitos y adquiridos pueden ejercer una acción sinérgica.

Existen una serie de tests que deben realizarse de forma sistemática cuando se sospecha un estado hipercoagulable (tabla I) (1).

Como orientación, indicar que la mayoría de los defectos congénitos van a provocar un riesgo aumentado de trombosis venosas, pero no arteriales.

En la población caucásica con trombosis venosa, los marcadores que van a estar afectados por orden de frecuencia son los siguientes:

1. Factor V de Leiden (12-40\%).

2. Homocisteína (10-20\%).

3. Mutación G20210A de la protrombina (6-18\%).

4. Deficiencias de antitrombina III, Proteína C y Proteína S (5-15\%).

5. Síndrome de anticuerpos antifosfolípidos (5$10 \%)$.

Respecto a las trombosis arteriales, los marcadores implicados más frecuentemente son los niveles de homocisteína en ayunas y los del síndrome de anticuerpos antifosfolípidos.

\section{PATOLOGÍAS OFTALMOLÓGICAS E HIPERCOAGULABILIDAD}

Existen patologías neuro-oftalmológicas o retinianas concretas en las que el estudio puede ser más selectivo, como expondremos a continuación.

\section{Obstrucciones venosas de la retina}

Las oclusiones venosas de la retina (OVR) se diferencian de otros tipos de trombosis venosas por su diferente patogénesis y factores de riesgo, el papel de la presión ocular (PIO) elevada, la delicada estructura de la retina, la ausencia de complicaciones embólicas y la ineficacia de los agentes fibrinolíticos.

Los factores implicados con más frecuencia en las OVR son hipertensión arterial, diabetes, hiperlipidemia, glaucoma, tabaco y aterosclerosis. La lipoproteína A puede ser hipofibrinogenolítica debido a que 
posee una secuencia de aminoácidos similar al plasminógeno, compitiendo en su lugar de unión con la fibrina e inhibiendo por tanto su degradación. Ésta puede ser una razón por la que la hiperlipidemia es un factor de riesgo para las OVR (5).

Estudios aislados y contradictorios han intentado establecer una relación de las OVR con los niveles de proteína $\mathrm{C}$ y $\mathrm{S}$, antitrombina III, factor V de Leiden/resistencia a la PCA, polimorfismo del gen G20210A del factor II (protrombina). En el momento presente, no hay evidencia suficiente para estudiar de forma sistemática estos factores en pacientes con OVR (5-8).

También se han encontrado niveles elevados de FAP-1 en pacientes con OVR comparado con sujetos normales. Además pacientes con OVR muestran un nivel de polimorfismo 4G del gen FAP-1 significativamente elevado [ $88 \%$ en pacientes con OVR comparado con un $63 \%$ en pacientes control $(\mathrm{p}=0,03)$ ], que se asocia a hipofibrinolisis, Aunque estos resultados son interesantes, de momento tampoco hay evidencia suficiente para recomendar el estudio sistemático del FAP-1 en pacientes con OVR $(5,9,10)$.

El papel de los anticuerpos antifosfolípidos parece ser más claro, especialmente en pacientes sin factores de riesgo conocidos. Además, la detección de anticuerpos antifosfolípidos tiene importantes implicaciones diagnósticas y terapéuticas: el diagnóstico de una posible enfermedad autoinmune o un síndrome antifosfolípido primario con la consiguiente indicación de anticoagulación y seguimiento estrecho de su situación inmunológica $(10,11)$.

También se han descrito elevaciones moderadas de homocisteína en plasma de pacientes con OVCR. La terapia vitamínica sería efectiva en reducir los niveles de homocisteína en este grupo de pacientes, pero su eficacia real en la prevención de nuevos episodios de OVCR no ha sido establecida (7).

Dada la baja prevalencia de muchas de estas raras enfermedades, muchos test podrían dar resultados anormales, tratándose de falsos positivos. Si un test es positivo debe consultarse con el hematólogo y confirmarse antes de realizar el diagnóstico o comenzar el tratamiento.

Recomendaciones finales de estudios de hipercoagulabilidad en OVR

- Anamnesis:

- Descartar historia familiar o personal de trombosis.
- Descartar causas secundarias de OVR (glaucoma por cierre angular, deshidratación severa, enfermedades inflamatorias de la retina, tortuosidad congénita de las venas retinianas).

- Exploraciones obligatorias:

- Tensión arterial.

- PIO.

- Hemograma completo, hemoglobina y perfil lipídico.

Cribado de Hipercoagulabilidad: Indicado en pacientes jóvenes sin ningún factor de riesgo conocido. No se deben realizar estos test de forma rutinaria en pacientes con factores de riesgo conocidos. En pacientes mayores, sin factores de riesgo conocidos, deben considerarse sólo estudio de homocisteína y anticuerpos antifosfolípidos (es poco probable que pacientes mayores sin ningún factor de riesgo debuten con una trombofilia primaria) (5).

Como resumen, los test de cribado de hipercoagulabilidad (tabla II) en OVR se realizarán en (5):

- Pacientes con OVR bilaterales y simultáneas.

- Menores de 50 años sin factores de riesgo identificables.

- Historia personal o familiar de trombosis.

\section{Oclusión arteria central de retina (OACR)}

Aparece típicamente en sujetos de edad avanzada, alrededor de la sexta década de la vida, de forma unilateral y con mayor prevalencia en el sexo masculino. En muchas ocasiones no es posible establecer el mecanismo patogénico exacto que conduce a la oclusión de la arteria central de la retina. Sin embargo, en la mayoría de los casos responde a alguno de los siguientes fenómenos: embolismo (lo más frecuente) (12), trombosis intraluminal, hemorragia bajo placa de ateroma, vasculitis, vasoespasmo, hipotensión arterial nocturna, aneurisma disecante y necrosis arterial hipertensiva.

Dada la elevada prevalencia de enfermedad aterosclerótica carotídea (45\%) asociada a la OACR, la realización de un eco-doppler de troncos supraaórticos está indicada de forma rutinaria en todos los pacientes (13). Un estudio eco-cardiográfico es necesario en sujetos con alto riesgo cardio-embolígeno (por ejemplo: prótesis valvulares), ausencia de patología carotídea, así como en todos los pacientes menores de 45 años (14).

Aunque las coagulopatías se relacionan típicamente con enfermedad oclusiva venosa, en sujetos 
Tabla II. Panel recomendado de pruebas de cribado de hipercoagulabilidad (1)

Panel rutinario

- Hemograma completo

- Función plaquetaria

- Prueba de fibrinógeno

- Electroforesis de proteínas séricas

- Tiempo de protrombina (TP), Tiempo parcial de tromboplastina (TTP)

- Pruebas de función hepática

- Función de Proteína C, Proteína S y Antitrombina III (con test para detección de mutación en el sitio de unión a la heparina)

- Resistencia a la Proteína C activada (Factor V de Leiden)

- Mutación de la Protrombina G20210A

- Anticuerpos antifosfolípido:

- Anticoagulante lúpico y Anticardiolipina (IgG, IgM)

- Si alta sospecha de síndrome antifosfolipídico: solicitar también anti-ß2 glicoproteína I

- Niveles de homocisteína (si los niveles son elevados: detectar niveles de folato, B12, B6 y mutación MTHFR)

- En sujetos de raza negra: Electroforesis de Hemoglobina

Pruebas a relizar si el panel inicial es negativo y persiste alta sospecha clínica de estado de hipercoagulabilidad

- Despistaje de coagulación intravascular diseminada

- Pruebas de disfibrinogenemia (tiempo de trombina, pruebas de función de fibrinógeno y tiempo de reptilasa)

- Pruebas para detección de actividad y antígeno del plasminógeno

- Cofactor II de la Heparina

- Sepsis

- Cáncer

Panel confirmatorio

- Tests inmunológicos (evaluación cuantitativa = actividad antigénica) de Proteína $\mathrm{C}$, proteína $\mathrm{S}$ y antitrombina III

- Repetir las pruebas de anticoagulante lúpico y anticuerpos anticardiolipina en 8 semanas

* Recordar que las pruebas de Proteína C, proteína S, antitrombina y anticoagulante lúpico deben realizarse antes de que se inicie la anticoagulación.

jóvenes (menores de 45 años), sobre todo aquellos menores de 30 años, el déficit de Proteina C y S así como de antitrombina III $(15,16)$ y la hiperhomocistinemia (17) pueden ser causa de oclusión arterial aguda.

Se han descrito algunos casos de OACR en pacientes con el Factor V de Leiden; sin embargo, dada la alta frecuencia de esta mutación en la población general la asociación en esos casos se considera fortuita. Tampoco la mutación G20210A de la protrombina parece jugar un papel relevante en sujetos por encima de los 45 años (18-20).

La presencia de anticuerpos antifosfolípido se ha relacionado con un cuadro de retinopatía isquémica crónica en la que la OACR o de rama es común. Aunque la búsqueda sistemática de estos marcadores inmunológicos no está bien establecida, existen estudios que apuntan una mayor frecuencia de los mismos en sujetos jóvenes (menores de 50 años) con cuadros vaso-oclusivos retinianos, así como en sujetos sin otros factores de riesgo $(11,21,22)$.

Por último señalar que la anemia de células falciformes puede complicarse con vasculopatía retiniana y algunos estudios han apuntado a que el origen primario sea una oclusión de arteria central de retina (23).

Resumen estudio hipercoagulabilidad en OACR

En paciente con OACR menor de 45-50 años, sin causa justificable debe realizarse cribado de enfermedades pro-trombóticas que incluya:

- Proteína C, S y Antitrombina III.

- Homocisteína.

- Electroforesis de hemoglobina (descartar enfermedad células falciformes en raza negra).

- Síndrome Antifosfolípido (Anticuerpo Anticardiolipina y anticoagulante lúpico).

\section{Neuropatía óptica isquémica anterior}

La neuropatía óptica isquémica anterior (NOIA) representa un evento isquémico multifactorial del nervio óptico. El factor desencadenante del cuadro suele ser una hipotensión arterial nocturna con descenso transitorio de la presión perfusión del nervio óptico hasta un nivel crítico (24) y no un fenómeno tromboembólico. 
Aunque en la literatura aparecen casos anecdóticos de NOIA en pacientes portadores de alteraciones de la coagulación, en el momento actual no hay argumentos suficientes para solicitar estudios de hipercoagulabilidad en estos pacientes.

\section{Pérdida visual transitoria}

Algunos pacientes con estados de hipercoagulabilidad pueden presentar episodios de pérdida visual transitoria (PVT). Normalmente es secundaria a una oclusión transitoria de la arteria central de la retina o de sus ramas y puede desarrollarse en asociación con cualquier anormalidad de la coagulación que produzca oclusión arterial. El síndrome de anticuerpos antifosfolípidos y la trombocitosis esencial son las causas más comunes (25). En más de la mitad de 50 pacientes con anticuerpos antifosfolípidos de un estudio retrospectivo se registraron alteraciones visuales transitorias de tipo visión borrosa, defectos parciales del campo visual y amaurosis fugax además de alteraciones visuales permanentes (26).

Glueck et al (27) estudiaron 19 pacientes con amaurosis fugax sin fuente embolígena detectable. Encontraron trastornos trombofílicos, como aumento de Factor VIII y IX, estado heterocigoto para protrombina G20210A, niveles reducidos de proteínas C y S, mutación MTHFR y PL A1/A2. También encontraron trastornos hipofibrinolíticos como aumento de lipoproteína A. Según la etiología los pacientes fueron tratados con cumarínicos, aspirina, enoxaparina, suplementos de vitamina B6, B12, ácido fólico, etc,.; consiguiendo así la desaparición de los síntomas visuales.

\section{Estudio de hipercoagulabilidad en PVT}

Ante una amaurosis fugax sin ateroesclerois carotídea u otra fuente embolígena se recomienda la búsqueda de trastornos de la coagulación, específicamente alteraciones relacionadas con oclusiones arteriales, fundamentalmente:

- Antitrombina III.

- Homocisteína.

- Síndrome Antifosfolípido.

- Electroforesis Hb (Enfermedad de células falciformes en raza negra).

La mayoría de estos trastornos son tratables.

\section{RECOMENDACIONES FINALES}

- Ante una sospecha de estado hipercoagulable debe realizarse un despistaje de factores de riesgo congénitos y adquiridos.

- Habitualmente se requiere la confluencia de múltiples factores de riesgo para que se desarrollen trombosis.

- En caso de resultados negativos y un elevado índice de sospecha se debe consultar a un hematólogo experto, que a menudo nos recomendará la solicitud de nuevos tests y nos dará indicaciones de tratamiento a largo plazo.

- Ante la presencia de factores congénitos es recomendable la evaluación y consejo genético.

- La presencia de factores de riesgo adquiridos puede potenciar el riesgo de trombosis preexistente, por lo que deben darse recomendaciones específicas en temas como contracepción, terapia hormonal de sustitución, embarazo, tabaco, etc.

\section{BIBLIOGRAFÍA}

1. Biousse V. Coagulation abnormalities and their neuroophthalmologic manifestations. Curr Opin Ophthalmol 1999; 10: 382-393.

2. Emmerich J, Rosendaal FR, Cattaneo M, Margaglione M, De Stefano V, Cumming T, et al. Combined effect of factor $V$ Leiden and prothrombin 20210A on the risk of venous thromboembolism-pooled analysis of 8 case-control studies including 2310 cases and 3204 controls. Study Group for Pooled-Analysis in Venous Thromboembolism. Thromb Haemost 2001; 86: 809-816.

3. Ardissino D, Mannucci PM, Merlini PA, Duca F, Fetiveau $R$, Tagliabue L, et al. Prothrombotic genetic risk factors in young survivors of myocardial infarction. Blood 1999; 94 : 46-51.

4. Den Heijer M, Rosendaal FR, Blom HJ, Gerrits WBJ, Bos GM. Hyperhomocysteinemia and venous thrombosis: a meta-analysis. Thromb Haemost 1998; 80: 874-877.

5. Lahey J, Kearney J, Tunc M. Hypercoagulable states and central retinal vein occlusion. Curr Opin Pulm Med 2003; 9: 385-392.

6. Greiner K, Peetz D, Winkgen A, Prellwitz W, Pfeiffer N, Hafner $G$. Genetic thrombophilia in patients with retinal vein occlusion. Int Ophthalmol 2001; 23: 155-160.

7. D'Angelo A, Tavola A, Fermo I, Mazzola G, Brancato $R$. Moderate hyperhomocysteinemia and central retinal vein occlusion. Thromb Haemost 2002; 87: 1078-1079.

8. Boyd S, Owens D, Gin T, Bunce K, Sherafat H, Perry D, et al. Plasma homocysteine, methylene tetrahydrofolate reductase C677T and factor II G20210A polymorphisms, factor VIII, and VWF in central retinal vein occlusion. $\mathrm{Br}$ J Ophthalmol 2001; 85: 1313-1315.

9. Marcucci R, Bertini L, Giusti B, Brunelli T, Fedi S, Cellai $A P$, et al. Thrombophilic risk factors in patients with cen- 
tral retinal vein occlusion. Thromb Haemost 2001; 86: 772-776.

10. Glueck CJ, Bell H, Vadlamani L, Gupta A, Fontaine RN, Wang $P$, et al. Heritable thrombophilia and hypofibrinolysis. Possible causes of retinal vein occlusion. Arch Ophthalmol 1999; 117: 43-49.

11. Cobo-Soriano R, Sánchez-Ramón S, Aparicio MJ, Teijeiro MA, Vidal P, Suárez-Leoz M, et al. Antiphospholipid antibodies and retinal thrombosis in patients without risk factors: a prospective case-control study. Am J Ophthalmol 1999; 128: 725-732.

12. Hayreh SS. Prevalent misconceptions about acute retinal vascular occlusive disorders. Prog Retin Eye Res 2005; 24: 493-519.

13. Shah HG, Brown GC, Goldberg RE. Digital substraction carotid angiography and retinal arterial obstruction. Ophthalmology 1985; 92: 68-72.

14. Sharma S, Sharma SM, Cruess AF, Brown GC. For the RECO Study Group. Transthoracic echocardiography in young patients with acute retinal artery occlusion. Can J Ophthalmol 1997; 32: 38-41.

15. Vignes $S$, Wechsler B, Elmaleh C, Cassoux N, Horellou $\mathrm{MH}$, Godeau P. Retinal arterial occlusion associated to activated protein C. Br J Ophthalmol 1996; 80: 1111.

16. Bertram B, Remky A, Arend O, Wolf S, Reim M. Protein C, protein $S$, and antithrombin III in acute ocular occlusive diseases. Ger J Ophthalmol 1995; 4: 332-335.

17. Wenzler EM, Rademakers AJ, Boers GH, Cruysberg JR, Webers CA, Deutman AF. Hyperhomocisteinema in retinal artery and vein occlusion. Am J Ophthalmol 1993; 115: 162-167.

18. Weger $M$, Renner W, Printer O, Stanger O, Temmel W, Fellner $P$, et al. Role of factor $V$ Leiden and prothrombin
$20210 A$ in patients with retinal artery occlusion. Eye 2003; 17: 731-734.

19. Tayyanipour R, Pulido JS, Postel EA, Lipkowitz JL, Pisciotta A, Braza E. Arterial vascular occlusion associated with factor V Leiden gene mutation. Retina 1998; 18: 376377.

20. Talmon T, Scharf J, Mayer E, Lanir N, Miller B, Brenner B. Retinal arterial occlusion in a child with factor $V$ Leiden and thermolabile - methylene tetrahydrofolate reductase mutations. Am J Ophthalmol 1997; 124: 689-691.

21. Levine SR, Crofts JW, Lesser GR, Floberg J, Welch KM. Visual symptoms associated with the presence of a lupus anticoagulant. Ophthalmology 1988; 95: 686-692.

22. Galetta SL, Plock GL, Kushner MJ, Wyszynski RE, Brucker AJ. Ocular thrombosis associated with antiphospholipid antibodies. Ann Ophthalmol 1991; 23: 207-212.

23. Lutty GA, Merges C, McLeod DS, Wajer SD, Suzuka SM, Fabry ME, et al. Nonperfusion of retina and choroid in transgenic mouse models of sickle cell disease. Curr Eye Res 1998, 17: 438-444.

24. Hayreh SS. Ischemic optic neuropathy. Prog Retin Eye Res 2009; 28: 34-62.

25. Winterkorn JM, Mack P, Eggenberger E. Transient visual loss in a 60-year-old man. Surv Ophthalmol 2008; 53: 301-305.

26. Leo-Kottler B, Klein R, Berg PA, Zrenner E. Ocular symptoms in association with antiphospholipid antibodies. Graefes Arch Clin Exp Ophthalmol 1998; 236: 658-668.

27. Glueck CJ, Golnik K, Ping W. Amaurosis fugax caused by heritable thrombophilia-hypofibrinolysis in cases without carotid atherosclerosis: thromboprophylaxis prevents subsequent transient monocular partial blindness. Clin Appl Thromb Hemost 2007; 13: 124-129. 